\title{
The Detuning Characteristics of Rational Harmonic Mode-Locked Semiconductor Optical Amplifier Fiber-Ring Laser Using Backward Optical Sinusoidal-Wave Injection Modulation
}

\author{
Gong-Ru Lin, Senior Member, IEEE, Member, OSA, Pai-Shen Hsueh, Hsiao-Hua Wu, and Yu-Sheng Liao
}

\begin{abstract}
The modulation waveform, power, and frequency detuning dynamics of a semiconductor optical amplifier fiber-ring laser (SOAFL) harmonic and rational-harmonic mode-locked by using backward injection of a large-signal sinusoidal-wave modulated Fabry-Pérot laser diode (FPLD) are studied. The shortest pulsewidth of $53.3 \mathrm{ps}$ at $1 \mathrm{GHz}$ with spectral linewidth of $0.09 \mathrm{~nm}$ at modulation power of $17 \mathrm{dBm}$ can be obtained, which corresponds to a time-bandwidth product of 2.8 . The threshold modulation power and the maximum frequency detuning range for harmonic mode-locking of the SOAFL are $11.5 \mathrm{dBm}$ and $180 \mathrm{kHz}$, respectively. The mode-locked SOAFL gradually recovers back to sinusoidal-wave modulation mode at the detuning frequency beyond $\pm 400 \mathrm{kHz}$. The pulse broadening slopes for the positive- and negative-frequency detuning regions of 0.48 and $0.375 \mathrm{ps} / \mathrm{kHz}$ are determined, respectively. By enlarging the modulating power and detuning the modulation frequency of FPLD, the SOAFL can be rational-harmonic mode-locked up to $12 \mathrm{GHz}$ with 33-ps pulsewidth.
\end{abstract}

Index Terms-Backward injection, cross-gain modulation, Fabry-Pérot laser diode, fiber-ring laser, frequency detuning, harmonic and rational-harmonic, mode-locking, semiconductor optical amplifier.

\section{INTRODUCTION}

$\mathbf{T}$ HE actively harmonic mode-locked travelling-wave semiconductor optical amplifier fiber-ring lasers (SOAFLs) or erbium-doped fiber amplifier based ring lasers (EDFLs) are the preferred sources to generate high-bit-rate pulses with relatively low timing jitter and high peak power at wavelengths of 1.3 and $1.55 \mu \mathrm{m}$ Typically, these actively mode-locked fiber-ring lasers can be implemented in terms of loss modulation using mode-lockers such as the Mach-Zehnder integrated-optic modulator [1], the electroabsorption modulator [2], and the Fabry-Pérot semiconductor modulator [3], [4]. These modulators not only increase additional loss inside the ring cavity but also introduce a limit on the operational bandwidth and make

Manuscript received January 12, 2004; revised October 18, 2004.

This work was supported by the National Science Council under Grants NSC-91-2215-E-009-039 and NSC-92-2215-E-009-028.

G.-R. Lin is with the Department of Photonics and Institute of Electro-Optical Engineering, National Chiao Tung University, Hsinchu, Taiwan 300, R.O.C. (e-mail: grlin@faculty.nctu.edu.tw).

P.-S. Hsueh and Y.-S. Liao are with the Graduate Institute of Optoelectronic Technology, National Taipei University of Technology, Taipei, Taiwan 105, R.O.C.

H.-H. Wu is with the Department of Physics, Tunghai University, Taichung, Taiwan 407, R.O.C.

Digital Object Identifier 10.1109/JLT.2004.839987 the system less cost-effective. Subsequently, gain modulation has emerged as another technique to achieve active mode locking in fiber-ring lasers. It can be carried out by directly modulating the injection current for the SOA or the pump beam for the EDFA. The former is a convenient approach to realize harmonic mode locking or even rational harmonic mode locking in fiber-ring lasers but still undergoes the electric bandwidth limitation of the SOA and additional bias network requirement. The latter is subject to the restriction of modulation bandwidth arising from long carrier lifetime of the EDFA. Based on the gain depletion effect, the gain modulation can also be attained by optical injection of a modulated signal to the gain medium. Recently, various new techniques have emerged to achieve harmonic mode-locking, rational-harmonic mode-locking [5], or frequency multiplication [6], [7] process in aforementioned lasers without the need of broadband optical modulating components. Later, the FM or AM mode-locking of SOA based fiber-ring lasers (SOAFLs) was demonstrated using intracavity Fabry-Pérot filter-based wavelength selector and filter.[8] In particular, the rational harmonic mode-locking at frequency up to $10 \times 30 \mathrm{GHz}$ can be achieved using a modulated distributed feedback laser diode (DFBLD) as seeding optical source and an intracavity filter as harmonic mode selector [9]. More recently, an all-optical actively mode-locked fiber ring laser based on cross-gain modulation in $\mathrm{SOA}$ at repetition frequency of $5 \mathrm{GHz}$ has been reported, which employs an externally modulated DFBLD as an optically controlled mode-locker [10].

In this paper, we study the detuning effect of a harmonic and rational-harmonic mode-locked SOAFL system using backward optical injection induced cross-gain-modulation technique. The gain of SOAFL can be periodically depleted by using a sinusoidal-wave modulated or gain-switched Fabry-Pérot laser diode (FPLD) at repetition of $1 \mathrm{GHz}$ as the backward seeding source. The effects of detuning the modulation waveform, power, and frequency on the mode-locking performances of the SOAFL are theoretically and experimentally characterized. The mode-locking range and the pulse broadening dynamics of the frequency detuned SOAFL are reported.

\section{EXPERIMENTAL}

The experimental setup of the $1.3-\mu \mathrm{m}$ fiber-pigtailed and backward FPLD-seeded SOAFL is shown in Fig. 1, which consists of a travelling-wave SOA, an FPLD, two Farady 


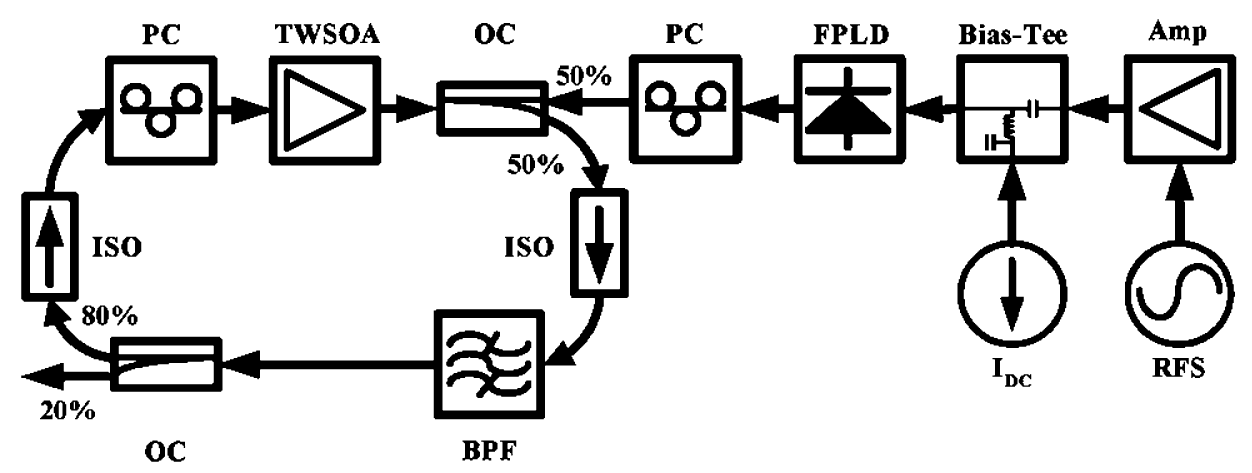

Fig. 1. Experimental setup of a harmonic mode-locked SOAFL using backward seeded FPLD as a gain modulation element. Amp: microwave amplifier; BPF: bandpass filter; FPLD: Fabry-Pérot laser diode; ISO: isolator; OC: optical coupler; PC: polarization controller; RFS: radio-frequency synthesizer; TWSOA: traveling-wave semiconductor optical amplifier.

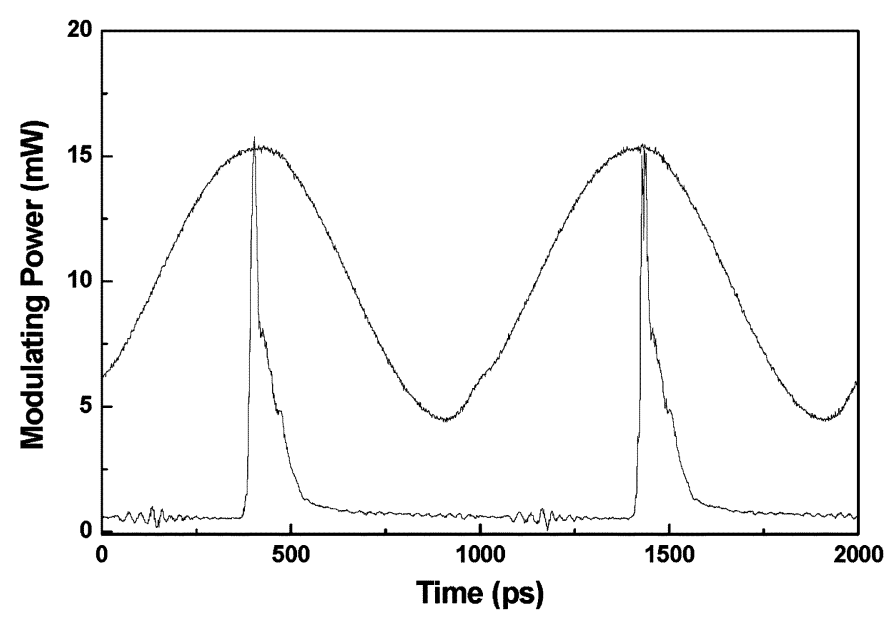

Fig. 2. Output traces of the FPLD sinusoidal-wave modulated (solid line) and gain-switched (dashed) at $1 \mathrm{GHz}$.

isolators, and two $1 \times 2$ optical couplers with various power splitting ratios. The SOA with maximum small-signal gain coefficient of $23 \mathrm{~dB}$ is dc biased at $70 \mathrm{~mA}$, which is slightly above threshold $\left(I_{\mathrm{th}}=60 \mathrm{~mA}\right)$ condition. The wavelength of peak gain and the $3 \mathrm{~dB}$ gain bandwidth of the free-running SOA are about 1306 and $31 \mathrm{~nm}$, respectively. The FPLD is sinusoidal-wave modulated at $1 \mathrm{GHz}$ using a radio-frequency (RF) synthesizer (Rohde \& Schwarz, SML01) in connection with a power amplifier of 24-dB gain. The linearly modulated FPLD is backward seeded into the SOAFL for cross-gain modulation (XGM) operation, which then induces harmonic mode-locking via the fine adjustment of the modulation frequency to match the one harmonic mode of the SOAFL. To ensure a linearly sinusoidal-wave modulation with higher modulation depth, the FPLD with longitudinal mode spacing of $93.8 \mathrm{GHz}(\Delta \lambda=0.75$ $\mathrm{nm}$ ) is dc biased from 35.6 to $58 \mathrm{~mA}$ as the RF signal amplitude increases from 8 to $19 \mathrm{dBm}$. Under such operation, the peak power of FPLD is increasing from 1 to $2.2 \mathrm{~mW}$, in which an average power from 0.15 to $0.35 \mathrm{~mW}$ is also observed. The maximum modulation depth of the FPLD is up to $80 \%$ or larger in this case (see Fig. 2). As the RF modulation power of the FPLD further increases beyond $21 \mathrm{dBm}$, the FPLD becomes gain-switched, as shown in Fig. 2. The FPLD is coupled into the closed-loop SOAFL ring cavity with coupling ratio of $50 \%$. The maximum output power of the SOAFL at contin- uous-wave mode (without the backward seeding of modulated FPLD signal) is $1.4 \mathrm{~mW}$. The Farady isolators are employed to ensure the unidirectional propagation of light and to avoid the amplification of backward seeded FPLD signal in the SOAFL ring cavity. The cavity length of the SOAFL is $21.16 \mathrm{~m}$, corresponding to a longitudinal mode spacing (i.e., fundamental repetition frequency) of $9.45 \mathrm{MHz}$. The harmonic mode-locking of SOAFL is achieved by matching the modulation frequency of FPLD to the one-hundred-sixth harmonics of the SOAFL cavity mode, which is about $1001.7 \mathrm{MHz}$. The mode-locked performances are characterized from the $80 \%$ coupling output of SOAFL by using a high-speed digital sampling oscilloscope $\left(\mathrm{HP} 86100 A+86109 B, f_{3 \mathrm{~dB}}>53 \mathrm{GHz}\right)$.

\section{RESULTS AND DISCUSSIONS}

\section{A. The Effect of Modulation Power Detuning}

The mode locking is achieved by injecting sufficient power of modulated laser diode to deplete and equivalently to modulate the gain in the SOA. The relation between mode-locked performance and modulated power of injection laser is therefore an important data to be built up. We found that the SOAFL cannot be harmonic mode-locked as the RF modulation power for the FPLD is less than $8 \mathrm{dBm}$. Meanwhile, the SOAFL exhibits small-signal sinusoidal and nonreturn-to-zero modulation. The gain-depletion of the SOAFL is not well established since the peak power of the linearly modulated FPLD is below $0.8 \mathrm{~mW}$. When the RF modulation power for the FPLD is between 8 and $11.5 \mathrm{dBm}$, we have observed weak and broadened optical pulses from the SOAFL ( $>150 \mathrm{ps})$ due to its insufficient gain-depletion depth. The threshold modulating power for FPLD to mode-lock SOAFL is around $11.5 \mathrm{dBm}$. As the modulation power of the FPLD increased from 12 to $17 \mathrm{dBm}$, the mode-locked pulse width is significantly reduced from 130 to 53.3 ps (see Fig. 3). The best RF amplitude and dc driving current of FPLD for mode-locking the SOAFL at shortest pulse width are $17 \mathrm{dBm}$ and $53 \mathrm{~mA}$, respectively. The shortest SOAFL pulse under the backward injection of sinusoidal-wave modulated FPLD is shown in Fig. 4. Theoretically, the mode-locked pulsewidth of the SOAFL can be estimated by [11]

$$
\tau=\sqrt{\frac{2 \ln 2}{\pi^{2}}} \cdot 4 \sqrt{\frac{2 g_{o}}{\Delta m}} \cdot \sqrt{\frac{1}{f_{m} \Delta \nu}}
$$




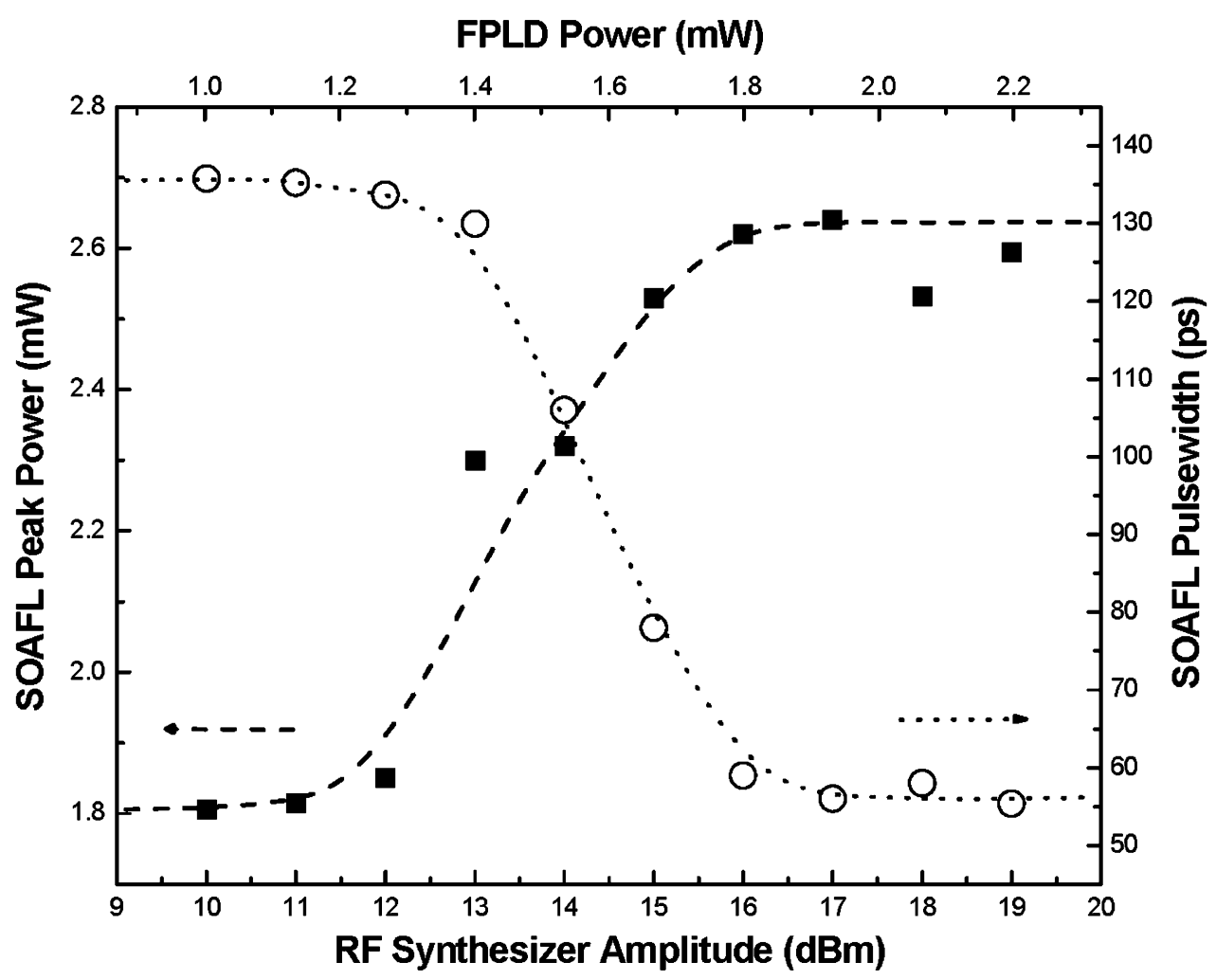

Fig. 3. Pulsewidth (hollow circle) and peak power (solid square) of the SOAFL at different of RF modulating powers and FPLD output powers.

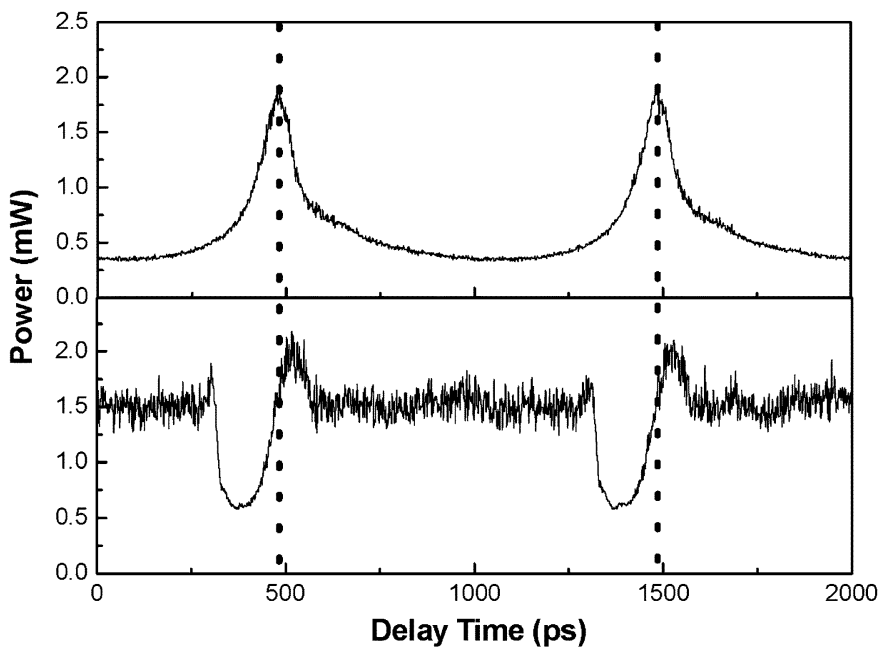

Fig. 4. Mode-locked pulses of SOAFL with the FPLD operating under sinusoidal-wave modulation (upper trace) and gain-switching (lower trace) conditions.

where $g_{o}$ denotes the round-trip gain of the SOAFL, $f_{m}$ denotes the modulation frequency of the FPLD, $\Delta \mathrm{m}$ is the measure of the modulation depth (on-to-off transmission ratio of the modulated FPLD), and $\Delta \nu$ is the gain bandwidth of the SOAFL. In our case, $g_{o}=22.5 \pm 0.5 \mathrm{~dB}, f_{m}=1001.7 \mathrm{MHz}, \Delta \mathrm{m}=4$, and $\Delta \nu=20 \mathrm{~nm} \cong 2.5 \mathrm{THz}$. The shortest pulsewidth of 53.3 ps from the harmonic mode-locked SOAFL at repetition frequency of $1 \mathrm{GHz}$ is somewhat larger than the estimated value of $30.8 \mathrm{ps}$, which may be attributed to the insufficient modulation depth and the excessive gain of the SOA. In addition, Fig. 5 illustrates the amplified spontaneous emission spectra of a free-run-

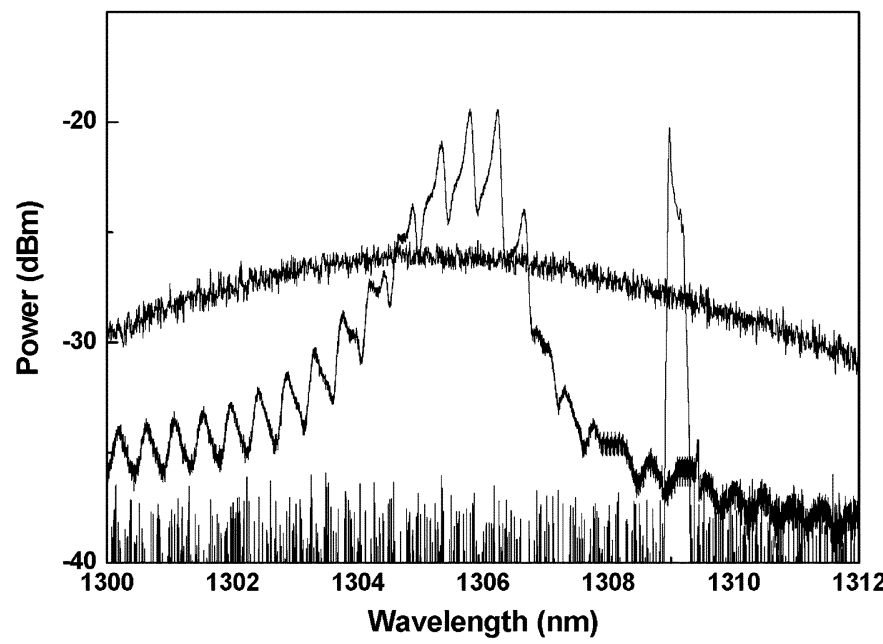

Fig. 5. Amplified spontaneous emission spectrum of the free-running SOA, and the lasing spectra of the sinusoidal-wave modulated FPLD and the FPLD backward-injection mode-locked SOAFL.

ning SOA at driving current of $50 \mathrm{~mA}$. The peak wavelength and $3 \mathrm{~dB}$ linewidth of gain spectra for the SOA driving at $40 \mathrm{~mA}$ are about 1304 and $20 \mathrm{~nm}$, respectively. The central wavelength of the sinusoidal-wave modulated FPLD is $1309 \mathrm{~nm}$. The lasing spectra of the SOAFL mode-locked by the FPLD modulating at different RF powers are shown in Fig. 6. The lasing threshold of the SOA is reduced when the SOA is closed-loop configured to form a SOAFL, which makes the cavity effect of the SOA more significant and reveals several longitudinal modes of the SOA with spacing of about $0.4 \mathrm{~nm}$ in the lasing spectrum. The peak wavelength of the FPLD mode-locked SOAFL is at $1306 \mathrm{~nm}$, 


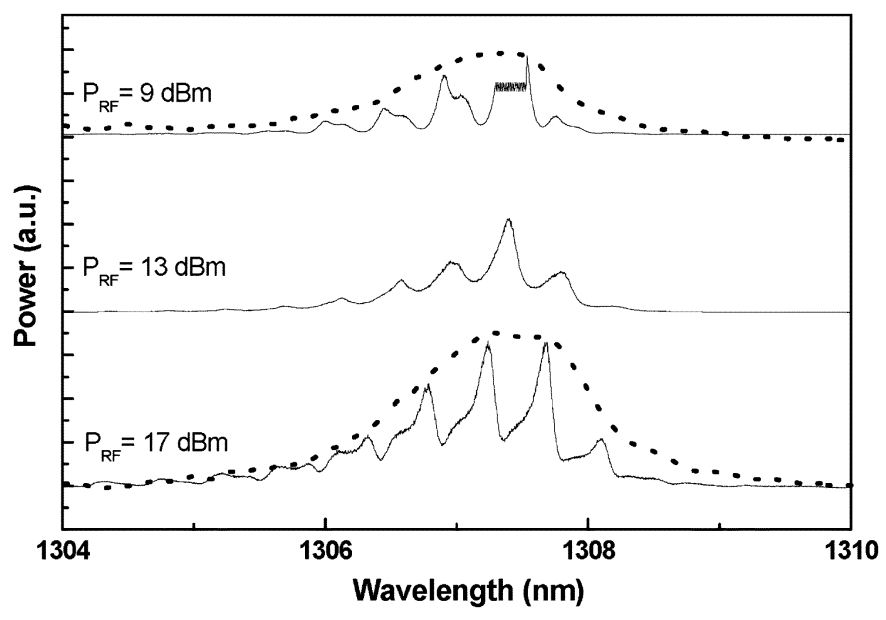

Fig. 6. Lasing spectra of the FPLD backward-injection mode-locked SOAFL with the FPLD at different modulating powers.

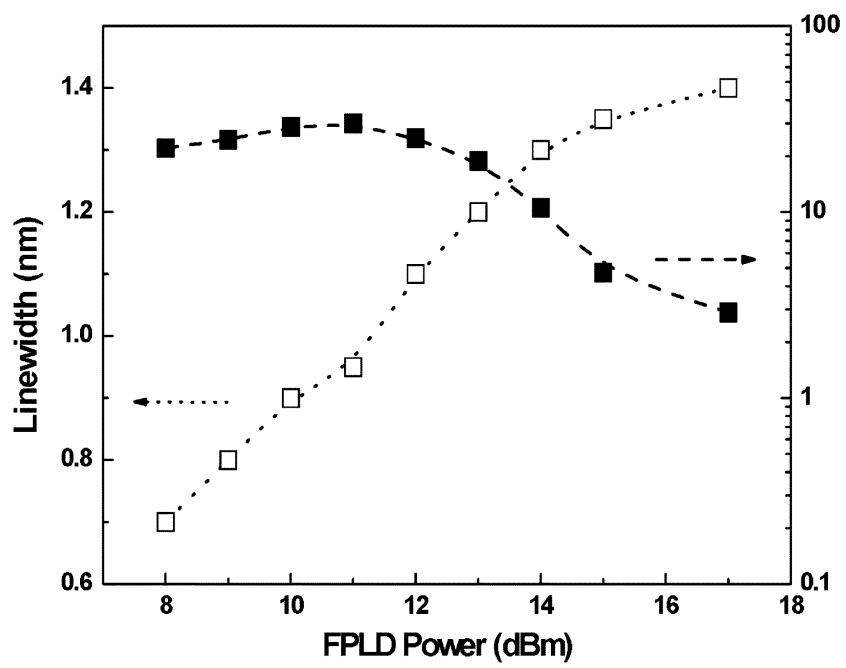

Fig. 7. Linewidth (hollow square) and time-bandwidth product (solid square) of the SOAFL at different of RF modulating powers.

which is slightly red-shifted due to the backward injection of DFBLD at $1309 \mathrm{~nm}$. The spectral linewidth of SOAFL pulses slightly broadens from 0.7 to $1.4 \mathrm{~nm}$ as the RF modulating power increases from 9 to $17 \mathrm{dBm}$. The lasing linewidth and the time-bandwidth product of the harmonic mode-locked SOAFL as a function of average power from the sinusoidal-wave modulated FPLD is shown in Fig. 7. The time-bandwidth product of 2.8 is obtained as the FPLD power increases up to $2 \mathrm{~mW}$.

The single-sideband (SSB) phase noise and timing jitter of the FPLD backward injection mode-locked SOAFL are comparable with those using conventional mode-locked schemes, as shown in Fig. 8. By using a frequency synthesizer with SSB phase noise density of $-120 \mathrm{dBc} / \mathrm{Hz}$ at offset frequency of $10 \mathrm{kHz}$ from carrier, the corresponding phase noise and timing jitter of the FPLD backward injection mode-locked SPAFL are determined as $-95 \mathrm{dBc} / \mathrm{Hz}$ at offset frequency of $10 \mathrm{kHz}$ from carrier and $0.3 \mathrm{ps}$ at integral region between $10 \mathrm{~Hz}$ and $10 \mathrm{kHz}$, respectively. A maximum side-mode suppression ratio of up to $40 \mathrm{~dB}$ has been observed. With a high-power sinusoidal-wave modulated FPLD, the SOAFL can easily be either harmonic or rational-harmonic mode-locked due to the sufficiently large gain-depletion

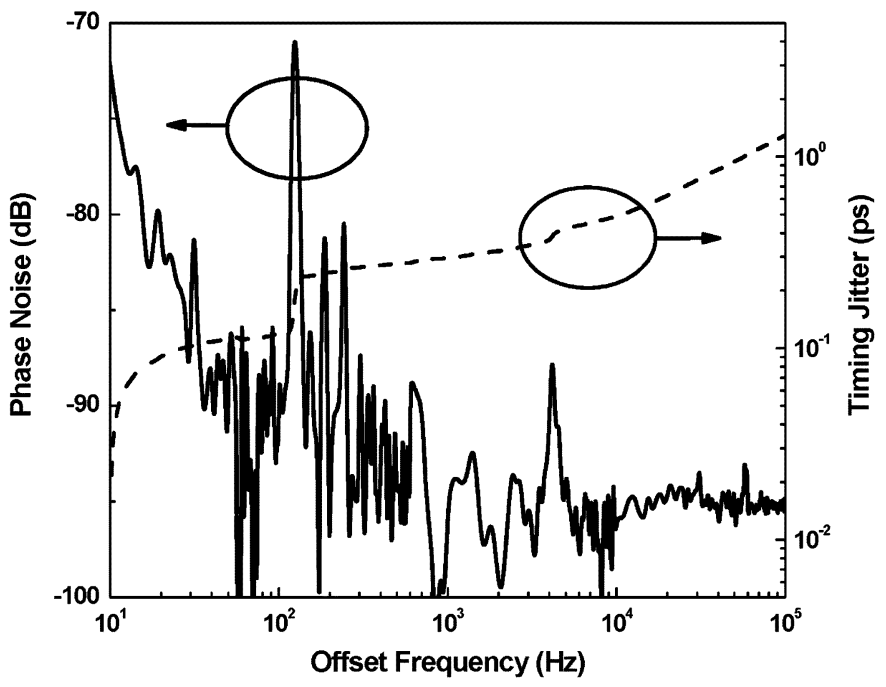

Fig. 8. The SSB phase noise spectrum and the corresponding timing jitter of the mode-locked SOAFL.

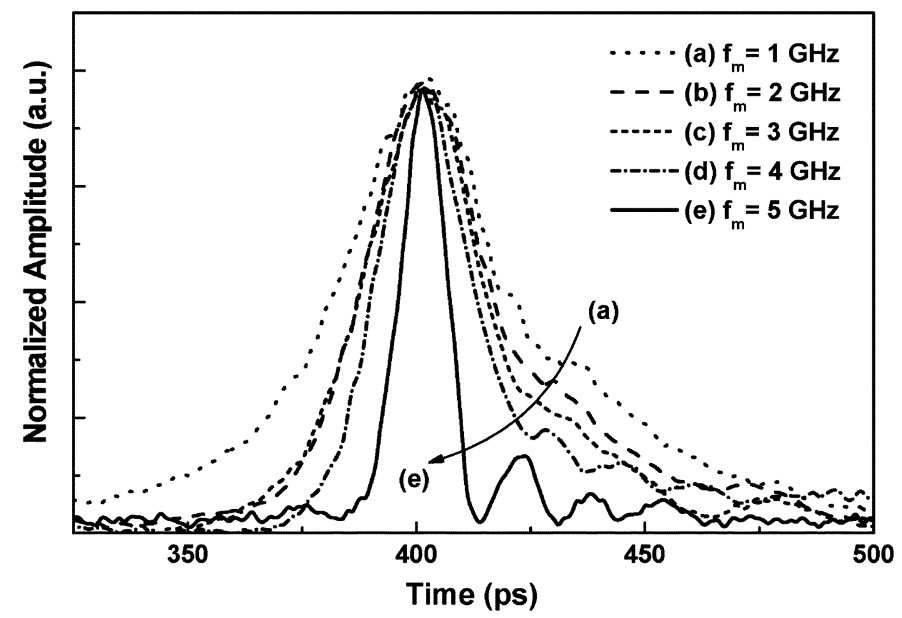

Fig. 9. The pulse shapes of the FPLD backward-injection mode-locked SOAFL at different harmonic mode-locking frequencies.

depth and narrow gain window. In addition, the pulse shapes of a FPLD harmonic mode-locked SOAFL at different repetition frequencies are illustrated in Fig. 9. The operating conditions of the FPLD, such as the RF modulating powers, the driving currents, and its output powers, etc., are listed in Table I. In experiment, the dc driving current of the FPLD remains constant and the RF modulation power is slightly increased to obtain a similar output power from FPLD. SOAFL pulsewidth as short as 12 ps can be obtained at harmonic mode-locking frequency of $5 \mathrm{GHz}$. The obtained pulsewidth is even shorter than those ever reported using a DFBLD in connection with a pulse-modulated integrated-optic modulator as the mode-locker [9]. On the other hand, the rational-harmonic mode-locking of SOAFL can also be achieved by slightly detuning the modulating frequency $\left(f_{m}\right)$ of FPLD apart from the harmonic mode frequency $\left(n f_{c}\right)$ of the SOAFL by $f_{c} / p$ to satisfy the condition of $f_{m}=(n+1 / p) f_{c}$, where $f_{c}$ is the fundamental SOAFL cavity frequency and $n$ and $p$ are integers denoting the harmonic and rational harmonic orders of the SOAFL, respectively. This leads to a frequency multiplication with repetition rate changing from $(n+1 / p) f_{c}$ to 
TABLE I

The Driving CONDITIONS OF FPLD AT DIFFERENT MODUlATING FREQUENCIES FOR HARMONIC MODE-LOCKING SOAFL

\begin{tabular}{cccc}
\hline Frequency & DC current & Modulating Power & Output Power \\
\hline $1 \mathrm{GHz}$ & $53 \mathrm{~mA}$ & $17 \mathrm{dBm}$ & $2.25 \mathrm{~mW}$ \\
$2 \mathrm{GHz}$ & $53 \mathrm{~mA}$ & $18.5 \mathrm{dBm}$ & $2.28 \mathrm{~mW}$ \\
$3 \mathrm{GHz}$ & $53 \mathrm{~mA}$ & $19.2 \mathrm{dBm}$ & $2.21 \mathrm{~mW}$ \\
$4 \mathrm{GHz}$ & $53 \mathrm{~mA}$ & $23.5 \mathrm{dBm}$ & $2.20 \mathrm{~mW}$ \\
$5 \mathrm{GHz}$ & $53 \mathrm{~mA}$ & $25.0 \mathrm{dBm}$ & $2.15 \mathrm{~mW}$ \\
\hline \hline
\end{tabular}

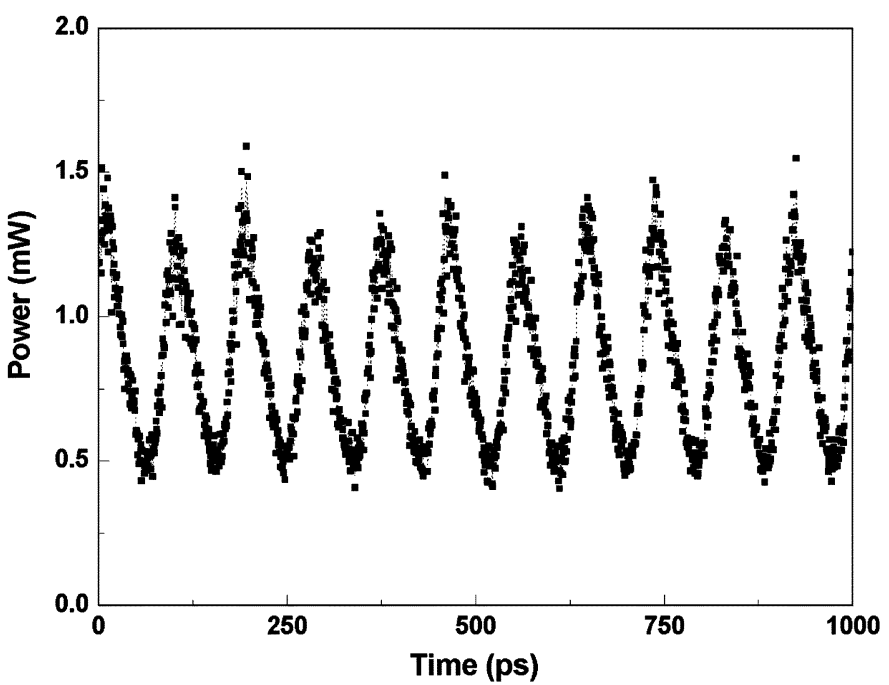

Fig. 10. The rational-harmonic mode-locked pulse-trace of SOAFL at repetition frequencies of $12 \mathrm{GHz}$.

$(n p+1) f_{c}$, which is exactly $p$ times the detuned modulation frequency of FPLD. As a result, the pulse-train of rational-harmonic mode-locked SOAFL with highest repetition frequency up to $12 \mathrm{GHz}$ (with $p=12$ ) is shown in Fig. 10. Note that the rational-harmonic mode-locked pulsewidth of SOAFL is about $33 \mathrm{ps}$, which is somewhat broadened and is not return-to-zero at such high repetition frequency. This is mainly due to the finite bandwidth of $f_{3 \mathrm{~dB}}=1 / 2 \pi\left(\tau_{p} \tau_{c}\right)^{0.5}$ of the SOA, where $\tau_{p}$ and $\tau_{c}$ are the photon and carrier lifetimes of the SOA.

\section{B. The Effect of Modulating Waveform Detuning}

The nonlinear modulation situation or gain-switching of FPLD appears as the RF modulating power of FPLD is further enlarged to $>21 \mathrm{dBm}$ or the dc current is decreased to threshold condition. The effect of backward injecting waveform on the transient gain of the SOA is illustrated in Fig. 11. A backward sufficiently large pulsewidth [or a sinusoidal-wave, see Fig. 11(a)] of the backward injection is essential to induce a wide gain-depletion region [and result in a narrow gain window, see Fig. 11(b)] of SOA for perfect mode-locking. The gain window is hereafter defined as the duration within one modulating period while the SOA still exhibits gain. In opposite, the backward injection of short optical pulse [see Fig. 11(c)] can only result in a less pronounced gain-depletion effect and induces a dip in the gain profile temporally. This causes a relatively wide gain window for the SOA, as shown in
Fig. 11(d). Such a wide gain window is helpless for generating short mode-locking pulses from SOAFL. Under the backward pulsed injection, a relatively broadened gain window of the SOAFL is left since the gain of SOA is not fully depleted within one modulation period, which consequently degrades the harmonic mode-locking pulses of the SOAFL. Note that a sufficiently narrow gain window is mandatory to the initiation of mode-locking process. To confirm, the output trace of SOAFL mode-locked by gain-switched FPLD with pulsewidth of 20 ps is shown in the lower trace of Fig. 4. In fact, the harmonic mode-locked SOAFL pulse width becomes broader (lengthening to $70 \mathrm{ps}$ or larger) at higher modulation powers $(>18 \mathrm{dBm})$ since now the FPLD turns to be gain-switching with a greatly decreased pulsewidth $(20 \mathrm{ps}$ at modulation power of $25 \mathrm{dBm}$ ). The peak power of the SOAFL at perfect mode-locking frequency is first increasing up to $2.7 \mathrm{~mW}$ at modulation power of $17 \mathrm{dBm}$, and then slightly decreases to $1.8 \mathrm{~mW}$ as the FPLD modulation power becomes larger. In comparison, the harmonic mode locking of SOAFL is relatively easy to establish via the optical injection from a sinusoidal-wave modulated FPLD instead of a gain-switched one. Note that the injection of a pulse-modulated or gain-switched FPLD signal at higher RF modulation powers only causes a narrower gain-depletion window. In frequency-domain analysis, the power in the fundamental frequency such a short-pulse modulated waveform is decreased, while the powers in the dc and higher harmonic components are gradually enlarged. This eventually leads to a weak mode-locked pulse followed by a larger pedestal, as shown in the lower trace of Fig. 4. Consequently, the SOAFL is continuous-wave lasing instead of harmonic mode-locking. Such a pedestal results from the insufficient gain depletion of SOAFL under the backward injection of gain-switched FPLD pulse.

To further investigate the effect of the backward injected waveform on the gain depletion and mode-locking performance of the SOAFL, the pulse shapes of harmonic mode-locked SOAFL gain-depletion modulated by sinusoidal-wave modulated and gain-switched FPLDs are theoretically simulated. By splicing the SOA in SOAFL into $M$ sections, the differential rate equations that describe the time-varied carrier density $\left(N_{j}\right)$ in the $j$ th gain section under the influence of mode-locked $\left(P_{1}\right)$ and backward injected $\left(P_{2}\right)$ signals with average powers of $\bar{P}_{1, j}$ and $\bar{P}_{2, j}$ are written as

$$
\frac{\partial N_{j}(z, t)}{\partial t}=\frac{I}{q V}-\frac{N_{j}(z, t)}{\tau_{c}}-\sum_{l} \frac{g_{l, j}(z, t)}{h v_{l} A_{\mathrm{cross}}} \overline{P_{l, j}}
$$




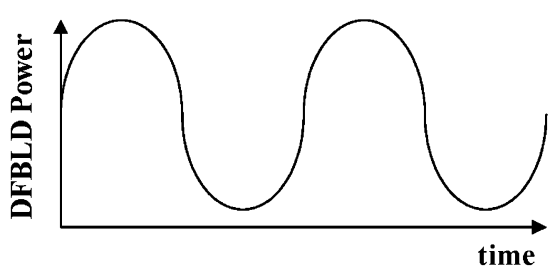

(a)

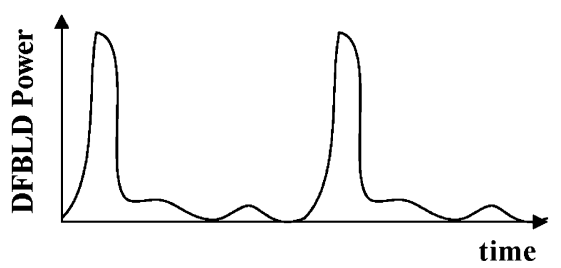

(b)

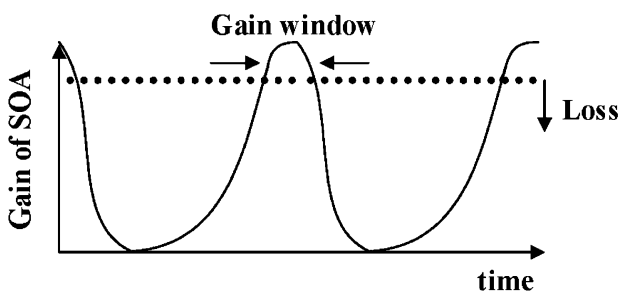

(c)

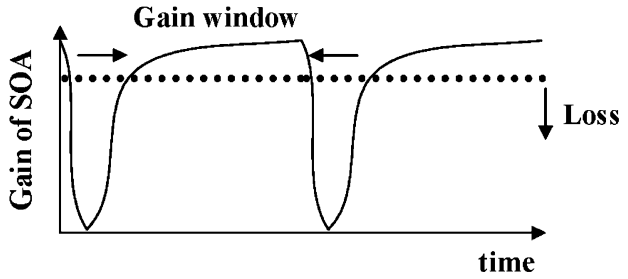

(d)

Fig. 11. The illustration of different gain windows in SOA caused by the different waveforms from the backward injected DFBLD.

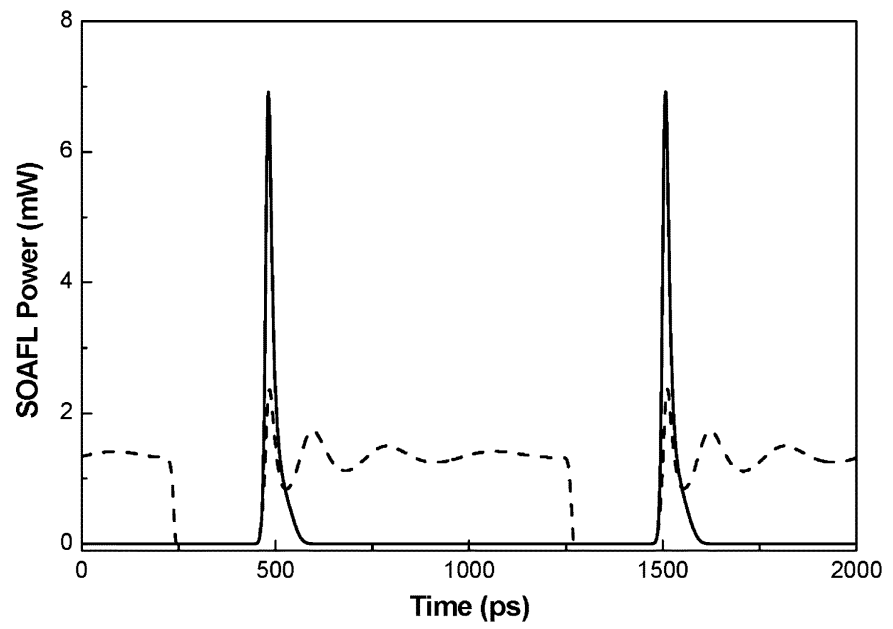

Fig. 12. The simulated pulse shapes of the harmonic mode-locked SOAFL with backward injection of sinusoidal-wave modulated (solid curve) and gain-switched (dashed curve) FPLDs.

$$
\begin{aligned}
\frac{\partial p_{l, j}(z, t)}{\partial z} & =(-1)^{l} \Gamma\left(g_{l, j}\left(N_{j}(z, t)\right)-\alpha_{\text {int }}\right) p_{l, j}(z, t) \\
\bar{P}_{l, j} & =\frac{1}{\Delta L} \int_{0}^{\Delta L} P_{l, j} e^{\left(g_{l}(\bar{N} j)-\alpha_{\text {int }}\right) z} d z \\
& =\frac{e^{\left[g_{l}\left(\bar{N}_{j}\right)-\alpha_{\text {int }}\right] \Delta L}-1}{\left[g_{l}\left(\bar{N}_{j}\right)-\alpha_{\text {int }}\right] \Delta L} P_{l, j} \\
\frac{\partial \phi_{l, j}}{\partial z} & =(-1)^{l-1} \frac{1}{2} \beta_{c} g_{l, j}\left(N_{j}(z, t)\right) \\
g_{l, j} & =a_{1}\left(N_{j}-N_{0}\right)-a_{2}\left(\lambda_{l}-\lambda_{N j}\right)^{2}+a_{3}\left(\lambda_{l}-\lambda_{N j}\right)^{3}
\end{aligned}
$$

where the index $1=1$ and 2 denotes the corresponding parameters for the mode-locked SOAFL signal and backward-injection modulated FPLD signals, respectively. In addition, $I, A_{\text {cross }}$, and $V$ denote the injection current, cross-section area in active area, and volume of SOA; $q$ is the electron charge; $h \nu_{l}$ denotes mode-locking and modulating photon energies; $\tau_{c}$ denotes the spontaneous emission lifetime; and $\alpha_{\text {int }}, g_{1}$, and $g_{2}$

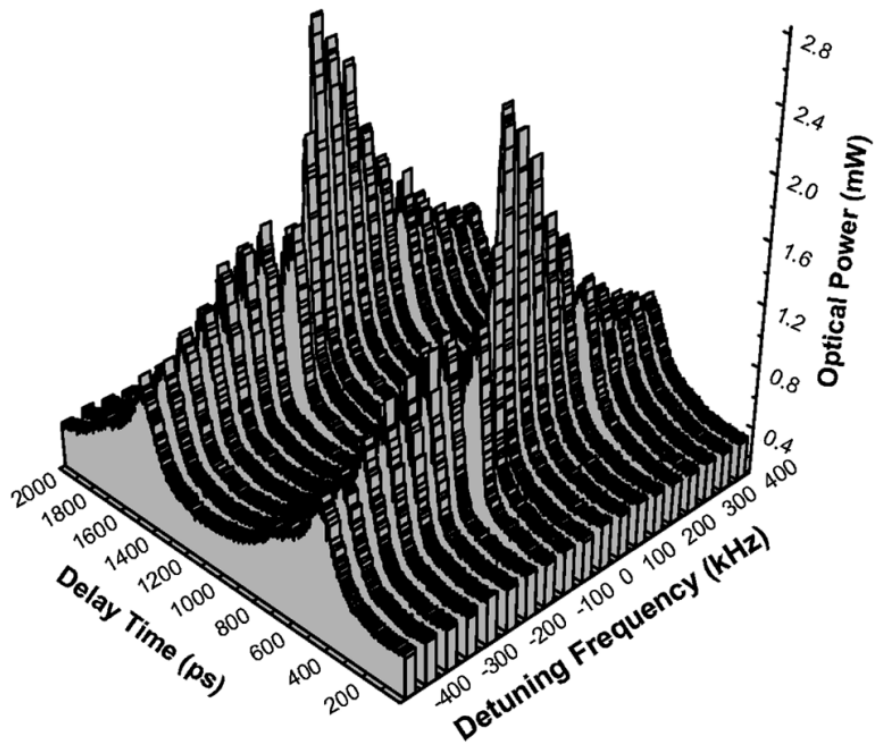

Fig. 13. The frequency-detuned pulse-train of the harmonic mode-locked SOAFL.

are the internal loss and the corresponding gain coefficients for the mode-locked and backward-injection modulated signals in SOAFL cavity, respectively. The spontaneous emission lifetime $\left(\tau_{c}\right)$ is obtained by $\tau_{c}^{-1}=A+B N_{j}+C N_{j}^{2}$ with $A, B$, and $C$ denoting the nonradiative, bimolecular, and auger recombination coefficients, respectively. Equation (6) is a cubic formula which simulates the gain coefficient of the DFBLD backward injection modulated SOAFL, in which $\alpha_{1}$ denotes the differential gain coefficient, $\alpha_{2}$ and $\alpha_{3}$ are experimentally determined constants which characterized the width and asymmetry of the gain profile, $N_{0}$ represents the transparent carrier density, $\lambda_{N}=\lambda_{0}-\alpha_{4}\left(N-N_{0}\right)$ is the corresponding wavelength for peak gain, and $\alpha_{4}$ denotes the empirical constant showing the shift of the gain peak. The parameters of the SOA used in our simulation are $\Gamma=0.3, \alpha_{\text {int }}=20 \mathrm{~cm}^{-1}, L=500 \mu \mathrm{m}$, $A_{\text {cross }}=0.4 \mu \mathrm{m}^{2}, \alpha_{1}=2.5 \times 10^{20} \mathrm{~m}^{2}, \alpha_{2}=7.4 \times 10^{18}$ $\mathrm{m}^{-3}, \alpha_{3}=3.16 \times 10^{25} \mathrm{~m}^{-4}, \alpha_{4}=3 \times 10^{-32} \mathrm{~m}^{4}, A=$ 


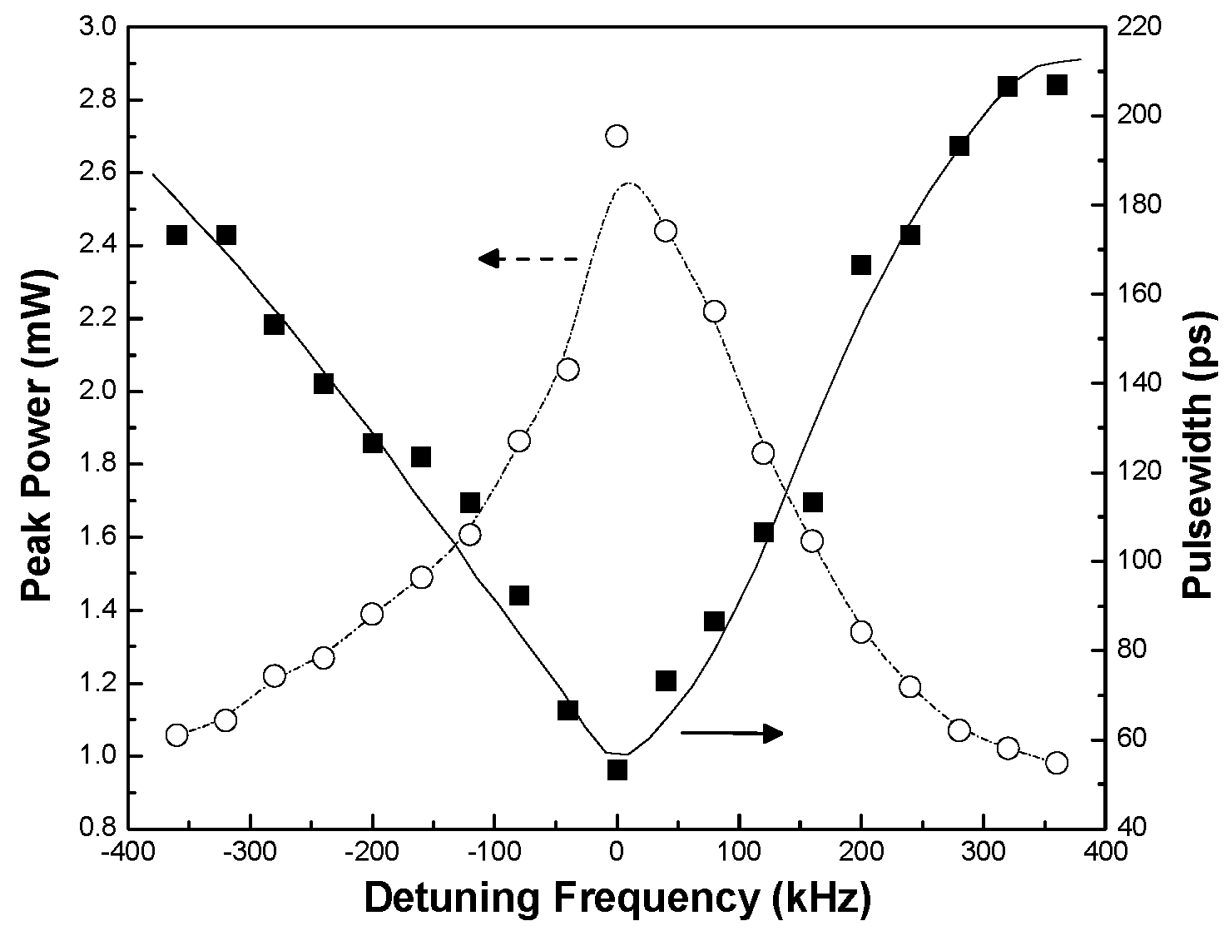

Fig. 14. The pulsewidth (solid square) and peak power (hollow circle) of the SOAFL at different detuning frequencies.

$2.5 \times 10^{8} \mathrm{~s}^{-1}, B=1 \times 10^{-16} \mathrm{~m}^{3} \mathrm{~s}^{-1}, C=0.94 \times 10^{-40}$ $\mathrm{m}^{6} \mathrm{~s}^{-1}, N_{0}=1.5 \times 10^{24} \mathrm{~m}^{-3}, q=1.6 \times 10^{-19} \mathrm{C}, c=$ $3 \times 10^{8} \mathrm{~m} / \mathrm{s}, \lambda_{0}=1309 \mathrm{~nm}, \lambda_{1}=1306 \mathrm{~nm}, \lambda_{2}=1306 \mathrm{~nm}$, $\beta_{2}=-20 \mathrm{ps}^{2} / \mathrm{km}, \gamma=10(\mathrm{~W} \cdot \mathrm{km})^{-1}, I=70 \mathrm{~mA}, P_{1}=$ $8 \times 10^{3}\left[1+0.9 \cos \left(2 \pi \nu_{1} t\right)\right]$, and $\nu_{l}=1 \mathrm{GHz}$. As a result, the continuous-wave lasing of SOAFL is more pronounced when compared with the small mode-locking peak in the rising part, as shown in Fig. 12. The continuous-wave lasing component (i.e., the pedestal after the principle pulse peak of the dashed line in Fig. 12) can be narrower when the pulsewidth of the gainswitched FPLD becomes wider, or which can be suppressed as the peak amplitude of the gain-switched FPLD becomes larger. The theoretical simulation reveals that such a pulse-modulated (or gain-switched) FPLD signal at higher RF modulation powers only causes an narrow gain-depletion window, which eventually leads to the degradation in mode-locked pulsewidth and peak power of the SOAFL. The lengthened high-level duration of the modulated FPLD signal (i.e., a larger duty cycle of the modulation), which can fully deplete the gain and temporally shrink the gain window, is mandatory to optimize the mode-locking performance of the SOAFL.

\section{The Effect of Modulating Frequency Detuning}

The evolution on the pulse shape of the harmonic modelocked SOAFL under the detuning of FPLD modulating frequency is shown in Fig. 13. The change in pulse shape is not significant within the detuning range of $\pm 10 \mathrm{kHz}$. The degradation in pulsewidth is less than $10 \%$ within detuning frequency of $\pm 50 \mathrm{kHz}$. Fig. 14 plots the measured output pulsewidth and peak power against the detuning frequency for the backward FPLD seeded, harmonic mode-locked SOAFL. The shortest pulsewidth of 53.3 ps associated with highest peak power of $2.7 \mathrm{~mW}$ for the SOAFL at perfect mode-locked frequency is observed. As the modulation frequency of FPLD detuned from zero to $\pm 400 \mathrm{kHz}$, the mode-locked pulsewidth is enlarged from 53.3 to $206.7 \mathrm{ps}$ and the peak power is decreased from 2.7 to $0.98 \mathrm{~mW}$. The locking range of the harmonic mode-locked SOAFL (which is defined as the bandwidth of detuning frequency which causes pulse broadening of 50\% from the original pulsewidth) is about $180 \mathrm{kHz}$. The mode-locked pulse is further degraded and becomes similar to sinusoidal wave at detuning frequency beyond $\pm 320 \mathrm{kHz}$, and the SOAFL finally transforms into sinusoidal-wave modulation mode as the detuning frequency beyond $\pm 400 \mathrm{kHz}$, which interprets that the mode-locking mechanism no longer exists in the SOAFL. The rational harmonic mode-locking phenomenon is not observed in the SOAFL output since the detuning frequency of $400 \mathrm{kHz}$ is corresponding to a thirty-fifth-order rational harmonic mode-locking at repetition frequency of $35 \mathrm{GHz}$.

Moreover, it is found that the effects of positive- and negativefrequency detuning process on the pulse shape distortion and pulsewidth degradation are completely different, as shown in Figs. 15 and 16. Under positive-frequency detuning, the pulseshape broadening in leading and trailing edges of the harmonic mode-locked SOAFL pulse shape remains almost identical. In contrast, the increasing falling time of the pulse shape under negative-frequency procedure is more pronounced due to the growth of a secondary pulse peak [see Fig. 4(b)]. It is easily understood that when the gain depletion process is delayed due to the negative-frequency detuning, the trailing edge of the modelocked pulse shifts across the peak and toward the rising part of the gain profile, which subsequently experiences higher gain than the leading edge. This inevitably leads to the broadening in trailing edge together with a slightly larger decaying rate in peak power of the harmonic mode-locked SOAFL pulse. The pulse broadening slope of the positive-frequency detuning region of 


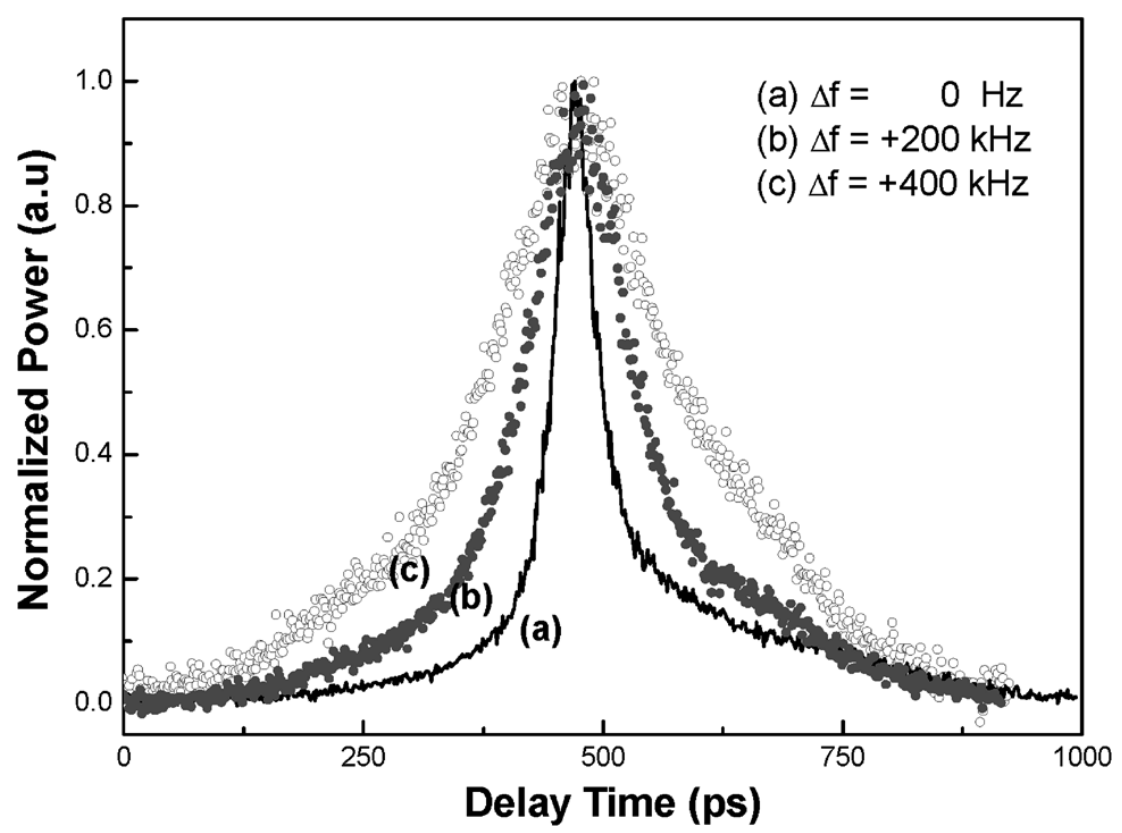

Fig. 15. The evolution of harmonic mode-locked SOAFL pulse shapes (a) without or with positively detuning frequencies of (b) $200 \mathrm{kHz}$ and (c) $400 \mathrm{kHz}$.

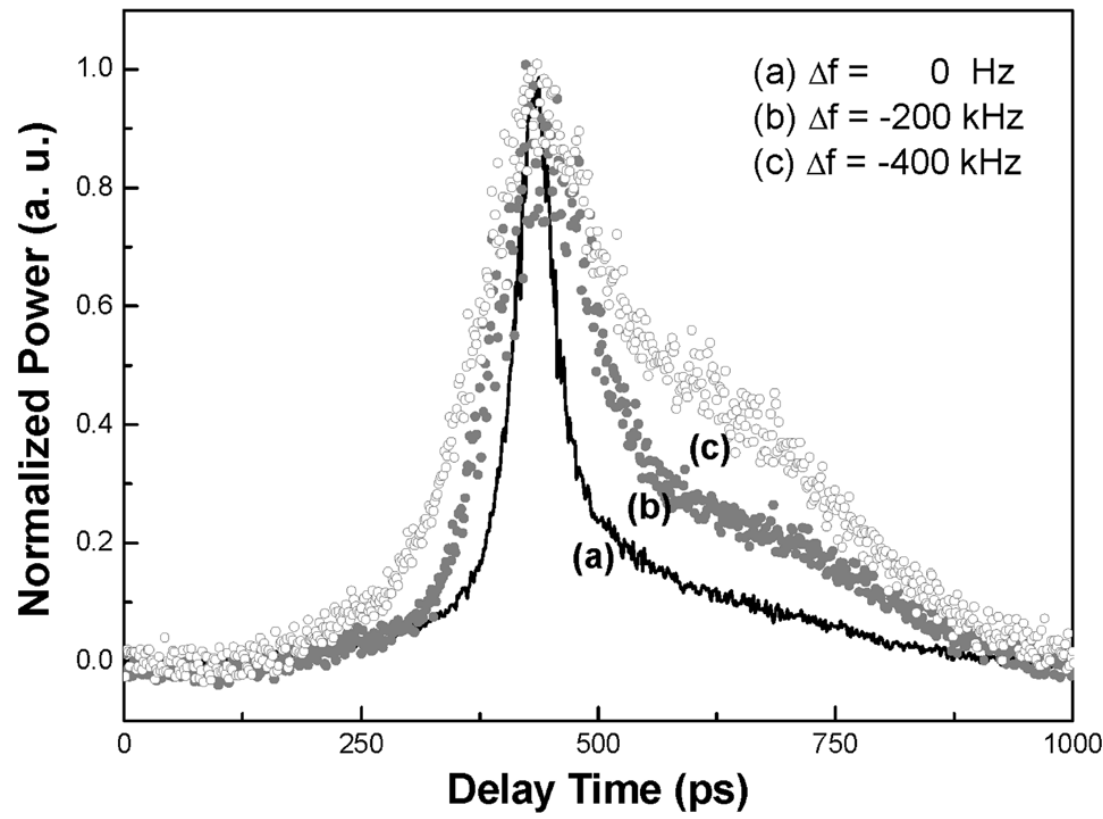

Fig. 16. The evolution of harmonic mode-locked SOAFL pulse shapes (a) without or with negatively detuning frequencies of (b) $200 \mathrm{kHz}$ and (c) $400 \mathrm{kHz}$.

$0.48 \mathrm{ps} / \mathrm{kHz}$ is slightly larger than that of the negative-frequency detuning region $(0.375 \mathrm{ps} / \mathrm{kHz})$. It seems that the broadening ratio of the negatively frequency detuned pulse is less distinct as compared to that observed in positive frequency detuning process. However, the actual pulsewidth of the negatively frequency-detuned harmonic mode-locked SOAFL is not reflected in Fig. 3(b), since the peak power of the incompletely separated secondary pulse appeared on the trailing edge is lower than half of the principle peak power.

\section{CONCLUSION}

The detuning dynamics of the backward optical injection induced harmonic and rational-harmonic mode-locking of
SOAFL using a sinusoidal-modulated or gain-switched FPLD seeding are reported. The effects of modulation waveforms, frequency, and power detuning on variation of pulse shape and pulsewidth of SOAFL are discussed. A backward seeded FPLD signal with larger duty cycle is mandatory to optimize the mode-locking performance of the SOAFL. The RF powers required for initiating and optimizing the mode-locking mechanism of SOAFL are 12 and $17 \mathrm{dBm}$, respectively. A nonlinear modulation of FPLD appears as the RF amplitude is further enlarged or the dc current is decreased, which can efficiently enhance rational-harmonic mode-locking frequency of the SOAFL up to $12 \mathrm{GHz}$. The mode-locked pulsewidth and peak power significantly degrade as the modulation power exceeds $18 \mathrm{dBm}$ or the modulation frequency detunes greater than 
$10 \mathrm{kHz}$ from the perfectly mode-locked condition. The maximum mode-locking frequency detuning range corresponding to a pulse broadening ratio of $50 \%$ is nearly $180 \mathrm{kHz}$. The mode-locked pulse further broadens up to $180-210$ ps with a decreasing peak power of $1.0 \mathrm{~mW}$, and gradually recovers back to sinusoidal-wave modulation mode at the detuning frequency beyond $\pm 320 \mathrm{kHz}$. The pulse broadening slope of the positive-frequency detuning region of $0.48 \mathrm{ps} / \mathrm{kHz}$ is slightly larger than that of the negative-frequency detuning region $(0.375 \mathrm{ps} / \mathrm{kHz})$. From these results, the higher stability of the mode-locked SOAFL under positive-frequency detuning condition can thus be concluded.

\section{REFERENCES}

[1] D. Pudo, L. R. Chen, D. Giannone, L. Zhang, and I. Bennion, “Actively mode-locked tunable dual-wavelength erbium-doped fiber laser," IEEE Photon. Technol. Lett., vol. 14, no. 2, pp. 143-145, Feb. 2002.

[2] M. J. Guy, J. R. Taylor, and K. Wakita, "10 GHz 1.9 ps actively modelocked fiber integrated ring laser at $1.3 \mu \mathrm{m}$," Electron. Lett., vol. 33, pp. 1630-1632, 1997.

[3] S. Li and K. T. Chan, "Actively mode-locked erbium fiber ring laser using a Fabry-Ṕerot semiconductor modulator as mode locker and tunable filter," Appl. Phys. Lett., vol. 74, pp. 2737-2739, 1999.

[4] S. Yang, Z. Li, X. Dong, S. Yuan, G. Kai, and Q. Zhao, "Generation of wavelength-switched optical pulse from a fiber ring laser with an F-P semiconductor modulator and a HiBi fiber loop mirror," IEEE Photon. Technol. Lett., vol. 14, no. 6, pp. 774-776, Jun. 2002.

[5] Z. Ahmed and N. Onodera, "High repetition rate optical pulse generation by frequency multiplication in actively modelocked fib," Electron. Lett., vol. 32, pp. 455-457, 1996.

[6] T. Pfeiffer and G. Veith, " $40 \mathrm{GHz}$ pulse generation using a widely tunable all-polarization preserving erbium fiber ring laser," Electron. Lett., vol. 29, pp. 1849-1850, 1993.

[7] K. K. Gupta and D. Novak, "Millimeter-wave repetition-rate optical pulse train generation in harmonically modelocked fiber ring laser," Electron. Lett., vol. 33, pp. 1330-1331, 1997.

[8] K. S. Abedin, N. Onodera, and M. Hyodo, "Repetition-rate multiplication in actively mode-locked fiber lasers by higher-order FM mode locking using a high-finesse Fabry-Pérot filter," Appl. Phys. Lett., vol. 73, pp. 1311-1313, 1998.

[9] K. Vlachos, K. Zoiros, T. Houbavlis, and H. Avramopoulos, "10 × 30 $\mathrm{GHz}$ pulse train generation from semiconductor amplifier fiber ring laser,' IEEE Photon. Technol. Lett., vol. 12, no. 1, pp. 25-27, Jan. 2000.
[10] J. He and K. T. Chan, "All-optical actively modelocked fiber ring laser based on cross-gain modulation in SOA," Electron. Lett., vol. 38, pp. 1504-1505, 2002

[11] N. V. Pedersen, K. B. Jakobsen, and M. Vaa, "Mode-locked $1.5 \mu \mathrm{m} \mathrm{semi-}$ conductor optical amplifier fiber ring," J. Lightw. Technol., vol. 14, no. 5, pp. 833-838, May 1996.

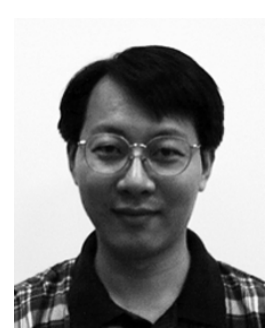

Gong-Ru Lin (SM'03) received the M.S. and Ph.D. degrees from the Institute of Electro-Optical Engineering, National Chiao Tung University, Taiwan, R.O.C., in 1990 and 1996, respectively.

$\mathrm{He}$ joined the Institute of Electro-Optical Engineering as an Associate Professor in 2002 and was promoted to Professor in 2004. His research interests are in ultrafast fiber lasers and optoelectronics, microwave and millimeter-wave photonics, and amorphous or nanocrystallite semiconductors. He has authored or coauthored more than 90 papers in international periodicals and more than 100 papers in international conferences. Dr. Lin is currently a Senior Member of the IEEE Lasers \& Electro-Optics Society (LEOS) and the Microwave Theory and Techniques (MTT) Society and is a Member of the Optical Society of America (OSA) and The International Society for Optical Engineers (SPIE). He has been included in Who's Who in Science and Engineering (6th ed.) since 2002 and was awarded the Tien Jea Bien Young Scholar Prize by the Optical Engineering Society of Republic of China for his outstanding achievement in photonics.

Pai-Shen Hsueh, photograph and biography not available at the time of publication.

Hsiao-Hua Wu, photograph and biography not available at the time of publication.

Yu-Sheng Liao, photograph and biography not available at the time of publication. 